\title{
La gestión de la crisis sanifaria de
}

la Covid-19 en España.

Efectos sobre la organización y funcionamiento del estade autonómice

\section{José María Pérez Medina}

Ex-Director General de Cooperación Autonómica y Local

E-mail: jmpmedina@miteco.es

DOI: 10.14422/ryf.vol285.i1455.y2022.001

Recibido: 8 de diciembre de 2021 Aceptado: 16 de diciembre de 2021

RESUMEN: La crisis del Covid-19 ha puesto de relieve la poca claridad del papel de la Administración del Estado y del Ministerio de Sanidad, en un sistema de reparto de funciones en el que el Estado carece de recursos asistenciales propios y, por lo tanto, corre el riesgo de un excesivo alejamiento de los problemas reales del sistema sanitario. La regulación normativa en vigor en materia sanitaria se ha mostrado insuficiente y puede requerir de previsiones normativas adicionales en caso de incumplimiento y que aseguren su efectividad.

PALABRAS CLAVE: Comunidades Autónomas; descentralización; sanidad pública.

\section{The management of the Covid-19 health crisis in Spain. Effects on the organization and functioning of the decentralized state}

ABSTRACT: The Covid-19 crisis has highlighted the lack of clarity in the role of the State Administration and the Ministry of Health, in a system of sharing functions in which the State lacks its own healthcare resources and, therefore, runs the risk of excessive distance from the real problems of the healthcare system. The current health regulations have proved to be insufficient and may require additional regulatory provisions to ensure their effectiveness in the event of non-compliance.

KEY WORDS: Autonomous Communities; decentralization; public health. 


\section{José María Pérez Medina}

\section{Introducción}

Desde hace ya casi dos años, en concreto desde el mes de marzo de 2020, los españoles hemos vivido de forma compartida un acontecimiento singular, excepcional, que hasta puede calificarse como insólito, y que ha supuesto unos cambios en la convivencia diaria y en el comportamiento social como nunca habíamos imaginado hasta ese momento.

Este proceso no puede compararse a ningún fenómeno o acontecimiento social conocido en décadas, pero lo cierto es que sus resultados han conmovido nuestra vida cotidiana y su recuerdo permanecerá durante décadas en la memoria colectiva: un estricto confinamiento de la población durante dos meses, en torno al 15\% de la población española directamente afectada y el doloroso dato de, cuanto menos, unos 88.000 fallecidos.

Los efectos de un acontecimiento tan extraordinario se han trasladado a todos los ámbitos de la vida colectiva, e incluso aún hoy continúan detectándose nuevos efectos, ahora en el ámbito económico con los problemas de desabastecimiento en el comercio mundial.

Y, lógicamente, los efectos de esta crisis también se han proyectado sobre la organización y funcionamiento del sistema político, y en singular el modelo autonómico.

Como punto de partida, una parte llamativa y muy visible de la opinión pública reprochó al modelo territorial de la complejidad de la gestión, añorando una mayor coordinación o, simplemente la unidad en la toma de decisiones, sin tener en cuenta las peculiaridades y las desigualdades territoriales de la incidencia de la crisis. Una realidad que aconsejaba un tratamiento específico desde la proximidad y el mejor conocimiento de cada territorio y de su componente social.

En todo caso, y frente a las críticas de la gestión autonómica, sí que puede argumentarse que la Covid-19 llevó a todos los poderes y responsables políticos a un nuevo escenario, calificado como de "gobernabilidad de lo impredecible", pues es obvio que ni el sistema político ni la organización territorial española se habían configurado pensando en una crisis similar.

\section{El sistema político ante la crisis sanitaria}

La crisis sanitaria rápidamente se convirtió en un examen para todo el sistema político y para la ges- 
tión de los tres niveles administrativos. En esta prueba, la necesidad de eficacia administrativa se situó automáticamente en el frontis de las decisiones y de las exigencias y ha marcado durante todo este periodo las relaciones entre el poder público y los ciudadanos.

Desde esta perspectiva, la crisis ha permitido una revisión del contrato social que vincula al Estado y a los ciudadanos, y ello en términos de actualización de lo que el Estado ofrece y espera y de lo que ciudadanos ceden y demandan al poder político.

En un primer momento, el Gobierno no duda en ejercer toda su autoridad y busca que los ciudadanos perciban su determinación para limitar las actividades de los particulares, incluso recurriendo cuando fuera necesario al uso de la fuerza coactiva y a cuantas medidas pudieran ser necesarias para hacer efectivas sus decisiones y garantizar su cumplimiento ${ }^{1}$.

Por este motivo, ya desde los primeros días quedó en evidencia una fundada preocupación por los derechos y libertades de los ciudadanos, pues medidas tan drásticas como los confinamientos domiciliarios, la suspensión

1 Real Decreto 4063/2020, de 14 de marzo. de la actividad económica o más recientemente la obligatoriedad de la vacuna, dibujan un panorama impensable en dos siglos de vida del sistema político liberaldemocrático. Nunca hasta ahora ningún Gobierno había sometido a sus ciudadanos a semejantes restricciones, aunque fuera con la justificada excusa de protegerlos de la expansión del virus, evitar los bloqueos de los dispositivos sanitarios $y$, en definitiva, salvar vidas humanas ${ }^{2}$.

En este marco, y al igual que en otros países, el Derecho vigente resultaba insuficiente para abordar una crisis de esta envergadura. Por ello, la crisis sanitaria de la Covid-19 plantea lo que se viene denominando "Derecho de necesidad". Es decir, invoca una regulación que con urgencia y determinación atienda un fenómeno tan complejo, incluso provocando una cierta ruptura o quiebra temporal y excepcional de las reglas jurídicas vigentes.

Y esta excepcionalidad ha afectado al poder público en todos sus niveles. Los principales efectos

\footnotetext{
2 El elemento más relevante de esta preocupación fue la Sentencia del Tribunal Constitucional 168/2021, de 14 de julio, sobre el primer estado de alarma; y la posterior Sentencia 183/2021, de 27 de noviembre.
} 


\section{José María Pérez Medina}

están a la vista de todos: el reforzamiento del poder ejecutivo; el papel secundario del legislativo, carente de información y criterios para poner en cuestión la estrategia del Gobierno; las dudas y heterogeneidad de interpretaciones en el Poder Judicial; la compatibilidad entre normativa sanitaria y de seguridad; la confusión entre responsabilidades de cada sector y cada nivel administrativo, etc. Y, desde luego, también ha afectado a la organización autonómica, básicamente porque la pandemia exige un punto de equilibrio entre la coherencia de las medidas que se toman a nivel nacional y las obligaciones asistenciales que corresponden a las Comunidades autónomas.

Pero, sobre todo, la crisis sanitaria conlleva una "exigencia de eficacia": la ciudadanía pide y exige a todas las Administraciones que no fallen en sus previsiones ni en sus medidas. $Y$ en este sentido, la crisis supone una actualización de la agenda del Estado: el Estado observa y se posiciona ante el ciudadano en una situación paradójica: el ciudadano es causa y difusor de la crisis, pero también es un individuo frágil que ante todo ha de ser protegido. Se sitúa así en un nuevo escenario, pero con la evidencia de que la búsqueda de la seguridad colectiva refuerza su legitimación y prestigio.

Además, el poder público despliega un amplio abanico de medidas de apoyo económico y social destinadas a los ciudadanos. $Y$ estas medidas, también refuerzan su posición atrayendo la atención y las reclamaciones de los sectores afectados por la crisis económica que acompaña al problema sanitario.

\section{Efectos para el funcionamiento efectivo del Estado Autonómico. Diversidad y pluralidad}

Desde la perspectiva de las Comunidades autónomas, la pandemia ha supuesto un test de idoneidad y una validación del sistema, al someterse a una prueba inédita hasta el momento.

Desde 1983, cuando se aprobaron los últimos Estatutos y se celebraron las primeras elecciones autonómicas, las Comunidades autónomas no se habían enfrentado a una situación comparable. Las decisiones trascendentales, aquellas que se relacionan con lo que en la actualidad se llaman "megatendencias": la globalización, la inmigración, el cambio climático, o la reconversión económica derivada del cambio económico global, han 


\section{La gestión sanitaria de la Covid-19 en España}

sido abordadas y gestionadas básicamente por el Gobierno central, incluso a veces en régimen de monopolio. Mientras tanto, las Comunidades autónomas actuaban como promotores, defensores de medidas e intereses territoriales, pero quedando siempre al margen de las decisiones finales que más afectan a los ciudadanos.

Pero la crisis causada por la Covid-19 es la primera ocasión en que los gobernantes autonómicos aparecen ante sus respectivas opiniones públicas como efectivamente responsables y dotados de una notable capacidad para decidir y condicionar la vida diaria de la población.

$Y$ en este sentido, y en nuestra opinión, la gestión autonómica de la crisis ha sido validada con cierto éxito, con las lógicas dudas y críticas, pero de forma globalmente satisfactoria. Las responsabilidades han sido atendidas, el sistema sanitario no ha colapsado en ningún momento y la cooperación entre profesionales de la sanidad ha funcionado de forma razonablemente satisfactoria. Ciertamente, esta afirmación puede ser cuestionada o matizada con la observación de detalles puntuales, pero también se dispone de argumentos y datos suficientes para sostener una valoración moderadamente positiva. Más adelante, el elevado y exitoso proceso de vacunación ha venido a completar la valoración general positiva que ha merecido la gestión de la crisis.

Además, los problemas planteados en España no han sido excesivamente diferentes de los detectados en otros Estados, incluso de organización centralizada, como es el caso de Francia, y en todos los Estados ha acabado por asumirse que la desigual incidencia y efectos de la crisis obligaba a soluciones diferentes en cada territorio.

En este sentido, los problemas y resultados obtenidos en España no han sido excesivamente diferentes. Incluso, es más, los niveles de aceptación y cumplimiento de las medidas impuestas han superado claramente los de la mayor parte de los Estados europeos ${ }^{3}$.

Esta aceptación ha resultado básica en el día a día al revalorizar la necesidad de complicidad social ante las medidas adoptadas; y esto ha cobrado especial relevancia en el caso de las Comunidades autónomas, sobre todo por la proximidad entre gobernantes y gobernados.

En la dosis correspondiente, la crisis supone un reconocimiento indirecto del principio de subsi-

3 Centro de Investigaciones SociolóGICAs. Estudios n. ${ }^{\circ} 3.279,3.283$ y 3.298 . 


\section{José María Pérez Medina}

diariedad, una asunción de que la cercanía del poder a la realidad avala que las decisiones sean tomadas en el nivel más próximo. Ahora bien, en contrapartida, la gestión de la crisis también ha hecho efectiva la responsabilidad de los gobernantes autonómicos, y no sólo del Gobierno central. Acostumbrados a remitir al nivel central de gobierno la responsabilidad por todos los problemas y a señalarlo como el último decisor de las políticas y medidas planteadas a los ciudadanos, en este caso la responsabilidad de los gobernantes aparece compartida, con un ineludible protagonismo de los Gobiernos autonómicos.

En esta relación gobernantesciudadanos, la pandemia ha reforzado los vínculos entre los ciudadanos y sus Comunidades autónomas. Ha favorecido la creación de espacios de debate de ámbito autonómico, propios, en los que se han contrastado las preocupaciones sanitarias y las demandas socioeconómicas de cada territorio. Esto ha supuesto un reconocimiento expreso del valor de la cercanía a la realidad social que ha de cumplir las indicaciones de los gobernantes. De esta manera, tras la sorpresa y demanda inicial de unidad de acción, la sociedad ha asumido la importancia de las peculiaridades de cada territorio y se ha ido asumiendo el principio general que postula que, a situaciones diferentes, corresponden soluciones diferentes.

\section{Equilibrios entre el poder de decisión del Gobierno y la capacidad gestora autonómica}

Los primeros pasos en la gestión de la crisis sanitaria no fueron especialmente sensibles a las atribuciones autonómicas, y el primer estado de alarma obvió sus competencias, atribuyendo al Gobierno la capacidad suficiente para disponer y dirigir los medios humanos y materiales de las Comunidades autónomas. Es decir, con el primer estado de alarma se pretendió una gestión única y centralizada de la crisis.

En un ambiente social de máxima preocupación, tanto el Gobierno como los ciudadanos buscan seguridad en la incertidumbre, y para ello incluso se exhiben los medios de autoridad que aportan las fuerzas y cuerpos de seguridad del Estado y el propio Ejército, incluso como medida de advertencia. A la vez, los responsables ministeriales se afanan en obtener la conformidad y colaboración social, y ello a pesar de las limitaciones de sus competencias. 


\section{La gestión sanitaria de la Covid-19 en España}

Sin embargo, este impulso inicial pronto se matizó, e incluso en buena parte cayó en el olvido. Por todo ello, puede decirse que la gestión de la Covid-19 ha ido modulándose desde un esquema calificable como centralista a otro plenamente descentralizado ${ }^{4}$. Este cambio no siempre ha sido comprendido por la opinión pública, y ha propiciado un ambiente de confusión y discrepancia política: primero se cuestionó el papel rector del Gobierno, pero más tarde se llegó a afirmar que el Gobierno se había desentendido de sus obligaciones en favor de las Comunidades.

En sentido estricto, la gestión de la crisis puede periodificarse distinguiendo cinco fases: una inicial, con medidas autonómicas propias (febrero 2020), el primer estado de alarma (marzo 2020), el levantamiento del estado de alarma y el denominado periodo de cogobernanza (junio 2020), el segundo periodo de estado de alarma (octubre 2020), y lo que podemos llamar normalidad actual (mayo 2021).

Estos cambios perfilan diferentes y sucesivos modelos de gobernanza que se visualizan al identificar a la "autoridad com-

4 Real Decreto-Ley 21/2020, de 9 de junio. petente". Primero lo fueron los miembros del Gobierno, y luego los Presidentes autonómicos, sobre todo cuando el Gobierno comprendió la imposibilidad del mando unificado.

Este cambio de actitud del Gobierno sin duda se correspondió con una postura más realista y menos ambiciosa, con un deseo de reconocer la pluralidad de situaciones en cada territorio, pero también se puede justificar como una respuesta lógica a la debilidad de sus apoyos parlamentarios en el Congreso y a su fuerte dependencia de otros grupos para que prosperaran sus medidas.

Sea por el motivo que sea, el reconocimiento de la realidad de cada territorio ha supuesto un incremento automático de las atribuciones autonómicas, pero también ha incrementado la complejidad y juego de equilibrios en la toma de sus decisiones, en especial conforme se evidencian las dudas e inquietud ante los efectos económicos de una ralentización de la actividad económica más prolongada de lo esperado.

Esta cuestión se fue infiltrando poco a poco en el argumento central de las discrepancias políticas y se erigió en dato imprescindible a la hora de tomar medidas, una vez comprobado como la crisis sanita- 


\section{José María Pérez Medina}

ria se convertía en pocas semanas en una seria crisis económica, con un fuerte retroceso de los indicadores de actividad y un incremento de la preocupación social.

Rápidamente, y cada vez con mayor intensidad, la atención a la salud tuvo que convivir con la inquietud por el deterioro de la realidad laboral. El resultado es un entrelazamiento entre salud y economía, en una relación causaefecto, que se retroalimenta mutuamente y que desborda la inicial y estricta atención a la salud de los ciudadanos.

Y en el ámbito autonómico, esta dualidad presenta especiales controversias, sobre todo por la cercanía de los actores sociales, profesionales y económicos y por sus posibilidades de ejercer mayor presión sobre las decisiones de los gobiernos.

En este marco, el papel de las Comunidades ha sido complejo y discutible, siempre a la búsqueda de un equilibrio que tuviera en cuenta simultáneamente los efectos sanitarios y económicos de las restricciones impuestas. $Y$ el resultado nunca ha estado exento de incoherencias, olvidos, decisiones precipitadas o, en definitiva, sospechas en la priorización.

La complejidad se acentúa aún más si se tiene en cuenta que los datos y las medidas para afrontar la situación sanitaria son escudriñadas casi a diario con atención. Esto supone que las medidas tomadas por las Comunidades están expuestas a una permanente comparación con las de otras, lo que no ha ayudado a la gestión sosegada y objetiva de la crisis.

\section{El Ejercicio de las Competencias Coordinadoras del Estado}

En el ámbito jurídico, la crisis ha supuesto recuperar el interés por la relevancia de las competencias coordinadoras del Estado. Se trata de un papel que en materia sanitaria de forma expresa la Constitución atribuye al Estado (art. 149.1.16 ${ }^{a}$, pero cuya efectividad práctica se remite a la legislación sanitaria posterior, en especial a la Ley General de Sanidad (1986), la Ley de Cohesión y Calidad del Sistema (2003) y la Ley General de Salud Pública (2011).

La regulación de la coordinación se justifica plenamente a partir de la jurisprudencia dictada por el Tribunal Constitucional, que ha precisado el alcance y contenido tanto de la cooperación como de la coordinación. El Tribunal concluye que la coordinación supone una 
posición de superioridad del Estado coordinador (STC 42/1983), pero $\sin$ que esto suponga una reconducción del papel de las Comunidades autónomas a una posición propia de entes subordinados (STC 71/2018). Y por ello, el Tribunal invita a la proporcionalidad en la coordinación.

No obstante, en esta jurisprudencia se detectan relevantes silencios, ya que el Tribunal no ha tenido la ocasión de explorar la forma de llevarla a la práctica, ni los efectos que puede suponer el incumplimiento de las medidas de coordinación o la simple imposibilidad de hacerla efectiva en determinadas situaciones. Como resultado, el sistema no garantiza el cumplimiento de las medidas de coordinación, y no siempre su cumplimiento es automático y ajustado a las medidas propuestas por el Gobierno.

La explicación de esta falta de atención es compleja y puede tener diferentes causas, pero es obvia la confianza, casi taumatúrgica, en la norma escrita y la certeza de que su mera existencia por sí sola supone una garantía de cumplimiento. Se trata de una visión excesivamente sobrevalorada por una lectura jurídica de las relaciones interadministrativas y que conduce al legislador a la conclusión de que la coordinación que- da asegurada de forma inmediata con la mera aprobación de la normativa básica estatal.

Desde una visión menos optimista, se aprecia cómo un importante déficit para la coordinación el hecho de que el Estado carezca de medios suficientes para garantizar el cumplimiento de las medidas coordinadoras que va aprobando. Y este déficit jurídico y material ha quedado en evidencia en la gestión de la crisis sanitaria.

Por otro lado, la jurisprudencia del Tribunal Constitucional ha ratificado tradicionalmente el papel voluntario de la cooperación, lo que probablemente tampoco ha ayudado a perfilar unos límites nítidos entre la coordinación y la cooperación y ha podido alimentar una interpretación excesivamente prudente de sus atribuciones por parte del Estado.

\section{El papel del Consejo Interterritorial del Sistema Nacional de Salud}

Quizás consciente de su débil posición, el Gobierno ha confiado especialmente en el papel del Consejo Interterritorial del Sistema Nacional de Salud, del que forman parte el Ministro y los 19 Consejeros autonómicos. Es más, podría decirse que en la prácti- 


\section{José María Pérez Medina}

ca la frecuencia de sus reuniones ha sustituido la falta de medidas coordinadoras obligatorias.

Ya en el momento de la crisis, en marzo de 2020, el Consejo era un órgano experimentado, con larga tradición en el sistema de cooperación y con una detallada organización técnica interna en diferentes niveles. Ahora bien, esa buena carta de presentación podía no ser suficiente para afrontar un reto como la nueva crisis, pues entre su acervo no figuraban antecedentes válidos para afrontar un problema de esta naturaleza y gravedad.

El hecho es que desde entonces el Consejo ha mantenido una intensa actividad, y ha pulverizado las estadísticas de sus reuniones, pues ha pasado de celebrar en torno a 4 reuniones anuales, a 82 en 2020 y a 51 en el periodo enero-noviembre de 2021.

Esta intensa actividad en el nivel superior del Consejo se complementa con una fructífera actividad de sus órganos técnicos y de apoyo. Esta es la aportación más valiosa del Consejo, la coordinación en el ámbito técnico, donde los expertos y mejores conocedores de la asistencia sanitaria acercan posturas e intercambian opiniones. En especial, es el caso de la Comisión de Salud Pública, la Ponencia de Vigilancia Epidemiológica o la Po- nencia de Alertas de Salud Pública. En este nivel inferior, el trabajo del Consejo ha sido continuado, discreto y ha aportado productos de utilidad, sobre todo diferentes documentos técnicos, que se han convertido en elementos de referencia para la actuación de las Comunidades y de los profesionales sanitarios.

Pero durante la gestión de la crisis, el Consejo ha tenido que afrontar también serias discrepancias sobre la obligatoriedad de sus acuerdos. Es decir, sobre la vinculación automática o no de sus decisiones para todas las Comunidades autónomas. La Ley de Régimen Jurídico del Sector Público, de 2015, establece con claridad esta obligatoriedad de los acuerdos de las Conferencias Sectoriales en su artículo 151, "cuando el Estado ejerce competencias coordinadoras", como es el caso de la política sanitaria, pero el vigente Reglamento del Consejo, que data de 2003, en su artículo 14 los cataloga como Recomendaciones que se adoptan por consenso. Esta discrepancia pone de relieve la falta de adaptación del Reglamento a la nueva Ley y, desde luego, cuestiona seriamente la efectividad de los acuerdos adoptados. Conociendo el marco de las relaciones políticas en España, estas dis- 
crepancias parecían inevitables, como su utilización en algunos momentos como instrumento para la oposición política.

Es así como la discrepancia jurídica ha derivado en conflicto y argumento para el reproche políti$\mathrm{co}$, originando un serio problema interpretativo sobre la naturaleza del Consejo, que ha llegado hasta el Tribunal Superior de Justicia de la Comunidad de Madrid y también a la Audiencia Nacional.

En todo caso, la utilización política del Consejo no debe ocultar su validez como foro de encuentro y punto de intercambio de información y posturas políticas en un momento de extrema presión para los responsables del sector.

El resultado más relevante de las decisiones políticas del Consejo son las diferentes "Declaraciones de Actuaciones Coordinadas", una nueva figura introducida en la Ley de Cohesión y Calidad del Sistema Nacional de Salud ${ }^{5}$ con el fin de intensificar el plan de acción conjunta, aunque los críticos más celosos de la autonomía política las han rechazado y llegado

5 Modificación introducida mediante el Real Decreto-Ley 21/2020, de 9 de junio. a considerar verdaderas "Órdenes comunicadas"6.

A pesar de la retórica empleada, el Consejo Interterritorial nunca ha sido ni es un órgano de cogobernanza del Sistema, ni siquiera un "órgano de dirección estratégica del Sistema"7, ya que no se trata de un órgano que tome decisiones conjuntas, de forma colegiada, y obligatorias para sus miembros, que sería la característica que permitiría considerarlo como tal. Más bien se trata de un órgano que ha actuado con una finalidad deliberativa y consultiva, previa a la posterior decisión del Estado, que se formaliza mediante la adopción de las medidas coordinadoras que competencialmente le corresponden y que se fundamentan en el art. 149.1.16 de la Constitución.

Es decir, en la práctica su actividad es doble: como "órgano de cooperación voluntaria" y como "órgano de consulta previa a la coordinación". Y por ello, estas dos visiones del órgano han generado confusión, hasta el punto de

\footnotetext{
6 Institut D'Estudis DE L'AUtOgOVERN. Generalitat de Catalunya. "Les relacions intergovernamentals en l'Estat autonòmic durant la crisi sanitària de la Covid-19", Informe 1 (2021).

7 Posibilidad ya prevista en la Recomendación $\mathrm{n}^{\circ}$ 4, del Informe Abril Martorell, de Reforma de la Sanidad. 1991
} 


\section{José María Pérez Medina}

trasladarse a los tribunales por la Comunidad de Madrid con la finalidad de que dirimiera el carácter obligatorio o no de sus acuerdos y, por lo tanto, la posibilidad de desligarse de los acuerdos que se iban adoptando.

\section{La polémica sobre la cogobernanza y la insatisfactoria actividad de la conferencia de presidentes}

Para justificar el cambio de la participación autonómica en la gestión de la crisis, el Gobierno se ha amparado en el reconocimiento e incorporación de las Comunidades autónomas al proceso de toma de decisiones, lo que se exhibe como un sistema o régimen de "cogobernanza", pero que fue cuestionado por un número relevante de Comunidades autónomas.

Esta mayor participación no exime, sin embargo, que las decisiones fundamentales en lo sustancial hayan sido tomadas por el Gobierno y trasladadas posteriormente a las Comunidades autónomas, lo que ha relegado la cogobernanza a una posición casi simbólica.

8 Diario de Sesiones. Senado. Comisión General de las Comunidades Autónomas. 30 de abril de 2020.
$\mathrm{Y}$ en este pretendido régimen de cogobernanza, la crisis sanitaria ha orientado el foco de la atención hacia la Conferencia de Presidentes, poniendo de relieve sus principales problemas: su débil institucionalización, la discontinuidad de sus reuniones, la falta de un método y agenda propia de trabajo, y la falta de una unidad ejecutiva que garantice su continuidad.

Igualmente se ha apreciado la dificultad para delimitar su ámbito de actuación material, que debiera ser aquel que excede de una determinada y única Conferencia Sectorial. Es decir, un ámbito de trabajo que se localice en lo supraterritorial y lo suprasectorial.

La falta de definición clara de estas dos cuestiones aleja a la Conferencia de Presidentes de otros modelos federales o incluso regionales equiparables y ha propiciado fundadas y sostenidas críticas a esta figura, sin que su expresa previsión en 2015, en la Ley de Régimen Jurídico del Sector Público, haya garantizado una mejora de su funcionamiento.

En especial, en este caso concreto, lo más preocupante ha sido la falta de reuniones del Comité Preparatorio, con lo que se ha incumplido reiteradamente su reglamento, en el que se atribuye a este órgano 
la preparación del Orden del día. Más bien al contrario, con motivo de las 18 reuniones celebradas desde el inicio de la crisis, se ha percibido un peso excesivo de los criterios propuestos por la Presidencia del Gobierno y una excesiva atención a sus prioridades y objetivos.

Durante este periodo, también se ha apreciado una manifiesta inacción para la adopción de medidas de apoyo económico y social que iban siendo tomadas tanto por el Gobierno como por las Comunidades, y sin prestar atención a evitar duplicidades, buscar complementariedad o simplemente dotar al debate político en la Conferencia de un contenido que fuera más allá del meramente sanitario.

\section{Conclusiones}

Con vistas al futuro, la crisis ha puesto de relieve la poca claridad del papel de la Administración del Estado y del Ministerio de Sanidad, en un sistema de reparto de funciones en el que el Estado carece de recursos asistenciales propios y, por lo tanto, corre el riesgo de un excesivo alejamiento de los problemas reales del sistema sanitario. Sobre todo, merece la pena recordar la escasez de los recursos humanos e incluso materiales con que cuenta.

En especial, se han echado en falta estructuras compartidas que desempeñen un trabajo de interés para todas las Administraciones, como corresponde al Instituto Robert Koch en la República Federal de Alemania, un órgano en cuyo funcionamiento efectivo participan los Länder. En especial, contrasta con el papel desempeñado por el Instituto de Salud Carlos III, que, lejos del carácter compartido del organismo alemán, es un organismo del Estado con escasa participación autonómica.

En estrecha relación con este punto, la experiencia de la crisis invita a revisar la legislación coordinadora del Estado. La regulación normativa en vigor en materia sanitaria se ha mostrado insuficiente y puede requerir de previsiones normativas adicionales en caso de incumplimiento y que aseguren su efectividad. El hecho de que las medidas coordinadoras sean previamente consultadas y debatidas con las Comunidades no obsta para que finalmente el Estado pueda hacer uso efectivo de sus atribuciones constitucionales.

En tercer lugar, el mejor rendimiento de la Conferencia de Presidentes requiere más que de cam- 
bios normativos, de un cambio de cultura política y administrativa que incremente el mutuo respeto y confianza, la mejor integración de posturas y una mayor autonomía de la Conferencia, al margen de la voluntad e intereses de la Presidencia del Gobierno, ya que la excesiva dependencia mostrada en la crisis le aleja de la condición de órgano de comunicación intergubernamental. 